\title{
Differential effects of lower body negative pressure and upright tilt on splanchnic blood volume
}

\author{
Indu Taneja ${ }^{1,2}$, Christopher Moran $^{2}$, Marvin S. Medow ${ }^{1,3}$, June L. Glover ${ }^{1}$, Leslie D. \\ Montgomery $^{1,4}$, and Julian M. Stewart ${ }^{1,3}$ \\ ${ }^{1}$ Department of Pediatrics, New York Medical College, Valhalla, New York \\ ${ }^{2}$ Department of Medicine, New York Medical College, Valhalla, New York \\ ${ }^{3}$ Department of Physiology, New York Medical College, Valhalla, New York \\ ${ }^{4}$ LDM, San Jose, California
}

\section{Abstract}

Upright posture and lower body negative pressure (LBNP) both induce reductions in central blood volume. However, regional circulatory responses to postural changes and LBNP may differ. Therefore, we studied regional blood flow and blood volume changes in 10 healthy subjects undergoing graded lower-body negative pressure $(-10$ to $-50 \mathrm{mmHg})$ and 8 subjects undergoing incremental head-up tilt (HUT; $20^{\circ}, 40^{\circ}$, and $70^{\circ}$ ) on separate days. We continuously measured blood pressure (BP), heart rate, and regional blood volumes and blood flows in the thoracic, splanchnic, pelvic, and leg segments by impedance plethysmography and calculated regional arterial resistances. Neither LBNP nor HUT altered systolic BP, whereas pulse pressure decreased significantly. Blood flow decreased in all segments, whereas peripheral resistances uniformly and significantly increased with both HUT and LBNP. Thoracic volume decreased while pelvic and leg volumes increased with HUT and LBNP. However, splanchnic volume changes were directionally opposite with stepwise decreases in splanchnic volume with LBNP and stepwise increases in splanchnic volume during HUT. Splanchnic emptying in LBNP models regional vascular changes during hemorrhage. Splanchnic filling may limit the ability of the splanchnic bed to respond to thoracic hypovolemia during upright posture.

\section{Keywords}

vasoconstriction; splanchnic; blood volume; orthostatic stress; hemorrhage

\begin{abstract}
Standing translocates blood from the central thoracic circulation to the dependent regional circulations, producing $20 \%$ reduction in cardiac output. The decrease in cardiac output comprises a $40 \%$ decrease in stroke volume associated with reflex tachycardia, increased peripheral resistance, and a generally maintained systolic blood pressure (SBP) with somewhat reduced pulse pressure (PP) while quietly standing (35). Changes in circulatory
\end{abstract}

Address for reprint requests and other correspondence: J. M. Stewart, Research Division and Hypotension Laboratory, New York Medical College, Ste. 3050, 19 Bradhurst Ave., Hawthorne, NY 10532 (stewart@ nymc.edu).. 
physiology during head-up tilt (HUT) are said to resemble the changes observed during hypovolemia caused by dehydration or hemorrhage $(39,41)$.

Lower body negative pressure (LBNP; see Refs. 44-46) has been used as a reversible simulation for hemorrhage $(8,30)$. LBNP has also been used to model orthostatic stress (7) because many of the changes of neurovascular physiology resemble changes during standing or HUT (31). Thus, for example, both HUT and LBNP produce central hypovolemia and comparable unloading of the cardiopulmonary and arterial baroreceptors $(9,19)$. However, gravitational differences in regional vascular properties have also been noted $(21,24)$. Thus recent work by Cooke et al. (8) and el Bedawi and Hainsworth (12) has demonstrated that, although LBNP physiology most closely resembles the physiology of acute hemorrhage, it may be incomplete as a model for orthostatic stress, which must produce changes in regional blood volume and blood flow that simulate standing.

Our recent (unpublished) work using impedance plethysmography (IPG) in the study of HUT demonstrated splanchnic filling (i.e., splanchnic hypervolemia; Fig. 1). Splanchnic filling during HUT differs from findings during hemorrhage in which splanchnic emptying occurs and may comprise up to $65 \%$ of the total volume of blood lost during the hemorrhage (17). Vatner (42), observing the central reservoir function of the splanchnic vasculature, noted that small increases in splanchnic arterial resistance sustained cardiac output during mild nonhypotensive hemorrhage, whereas others observed that incrementally severe hemorrhage produced almost complete splanchnic emptying $(5,37)$ by the rapid mobilization of venous blood in response to baroreceptor unloading $(18,32)$.

We hypothesize that LBNP resembles hemorrhage with a decrease in splanchnic volume, whereas splanchnic volume increases during HUT and is therefore directionally opposite to changes during LBNP. We propose that these differences in splanchnic volume changes occur despite similar changes in regional vasoconstriction, including splanchnic vasoconstriction, and those differences are primarily attributable to the effects of gravity during true orthostatic stress.

The current study was designed to compare and contrast regional circulatory blood flow, arterial resistance, and blood volume changes in healthy volunteer subjects undergoing LBNP and in healthy volunteer subjects undergoing HUT.

\section{METHODS}

\section{Subjects}

We recruited 18 healthy subjects (18-30 yr) divided into two groups that were tested on different days using LBNP $(n=10)$ and HUT $(n=8)$. The study was approved by the committee for the protection of human subjects (Institutional Review Board) of New York Medical College. Informed consent was obtained from all participants.

Subjects were recruited from a volunteer database and were screened for health status after a review of their medical history. Subjects were excluded if they were taking cardioactive or neuroactive medications. All procedures were performed after an overnight fast. Subjects 
refrained from beverages containing xanthine and caffeine for at least $72 \mathrm{~h}$ before testing.

All subjects were nonsmokers. We excluded volunteers with a history of syncope or orthostatic intolerance. There were no trained competitive athletes or bedridden subjects.

\section{Details of Method}

Heart rate and blood pressure monitoring-A single electrocardiogram lead was recorded for rhythm. Upper-extremity blood pressure (BP) was continuously monitored with a finger arterial plethysmograph (Finometer; FMS, Amsterdam, The Netherlands) placed on the right middle or index finger. Electrocardiogram and Finometer pressure data were interfaced to a personal computer through an analog-to-digital converter (DI-720; DataQ, Milwaukee, WI). Heart rate (HR) was derived from arterial pressure data. Finometer data were calibrated to a brachial artery oscillographic pressure. All data were multiplexed with impedance data and were thereby synchronized. Mean arterial pressure (MAP) was calculated from SBP and diastolic blood pressure $(\mathrm{DBP})$ from the formula $\mathrm{MAP}=(\mathrm{SBP}+2$ $\times$ DBP $) / 3$. PP was calculated from the formula PP $=$ SBP - DBP.

Changes in regional blood volumes-IPG using a tetrapolar high-resolution impedance monitor four-channel digital IPG (UFI, Morro Bay, CA) was used to measure changes in segmental blood volumes and segmental blood flows $(25,26)$. These quantities were obtained within four anatomic segments operationally defined by electrode placement on anatomic landmarks that delimit respective regional circulations. These were designated the thoracic segment (supraclavicular area to xyphoid process), the splanchnic segment (xyphoid process to iliac crest), the pelvic segment incorporating lower pelvis to the knee (iliac crest to knee), and the leg or calf segment (upper calf just below the knee to the ankle). $\mathrm{Ag} / \mathrm{AgCl}$ electrocardiogram electrodes were attached at these segmental boundaries and also to the left foot and left hand, where they served as current injectors. Electrical resistance values were measured by using the segmental pairs as sampling electrodes. The midline distance between the sampling electrodes $(L)$ was measured with a tape measure. We also measured the circumferences of calf, thigh, hips, waist, and chest to obtain approximate volume contents of each anatomic segment. We estimated postural changes in blood volume in each segment during HUT from the formula (15):

$\Delta$ segmental blood volume $(\mathrm{ml})=\rho \times\left(L^{2} / R_{0} R_{1}\right) \times \Delta R$

where $\rho$ is electrical conductivity of blood estimated as $53.2 \times \exp (0.022 \times$ hematocrit $)$ given by Geddes and Baker (14). $R_{0}$ is the resistance of a specific segment before change in HUT angle or degree of pressure, $R_{1}$ is the resistance after change in the HUT angle or degree of pressure, and $\delta R$ is the change in resistance $\left(R_{1}-R_{0}\right)$ in a specific segment during each incremental HUT or LBNP step; $\rho$ was regarded as constant during all maneuvers.

Blood flow-IPG was also used to measure segmental blood flows (27). Methods have recently been validated against the reference standard indocyanine green dye technique in our laboratory for the detection of leg, thoracic, and splanchnic blood flow $(38,40)$. Pulsatile changes in electrical resistance for each segment were employed to compute the time derivative $\partial R / \partial t$, which we used to obtain the blood flow responses of each body 
segment during HUT. Blood flow was estimated for an entire anatomic segment from the formula (14):

flow $=\left[\mathrm{HR} \times \rho \times L^{2} \times T \times \partial R / \partial t_{\max }\right] / R_{0}^{2}$

where $T$ is the ejection period, $R$ is the pulsatile resistance, and $R_{0}$ is the baseline resistance. Respiratory artifact was removed from the signal by using a custom, Fourier-based filtering technique. IPG flows were expressed in milliliters per minute for each anatomic segment and could be normalized by dividing by estimated segmental volume.

Peripheral resistance-Peripheral resistance was calculated as MAP/flow.

LBNP-Subjects were placed in the supine position such that their lower bodies (legs and hips up to the level of iliac crest) were placed in a sealed airtight chamber (the LBNP tank). The subject was sealed at the level of the iliac crest with a rubber diaphragm hermetically fitted around the iliac crest but not compressing the abdomen. Wire connections for impedance and venous plethysmography and tubes for cuff inflation were externalized through airtight ports. Suction was provided by a vacuum pump capable of rapidly producing desired negative pressure within 5-10 s and controlled with a variable autotransformer calibrated against an electronic manometer. The pressure (in Torr) was fixed by a pressure transducer. Graded LBNP was applied sequentially at $-10,-20,-30,-40$, and -50 $\mathrm{mmHg}$ for $5 \mathrm{~min}$ at each stage or until presyncope was achieved or the protocol was complete. Presyncope was defined as a decrease in SBP to $<80 \mathrm{mmHg}$; a decrease in SBP to $<90 \mathrm{mmHg}$ associated with symptoms of lightheadedness, nausea, or diaphoresis; or progressive symptoms of presyncope accompanied by a request from the subject to discontinue the test (13).

HUT table testing-An electrically driven tilt table (Colin Medical, San Antonio, TX) with a footboard was used. After supine measurements were complete, the table was tilted up sequentially (graded HUT at $20^{\circ}, 40^{\circ}$, and $70^{\circ}$ ) and maintained at each stage for $10 \mathrm{~min}$.

\section{Protocol}

Tests began after an overnight fast. Subjects were familiarized with the procedure used in the study. After a 30-min acclimatization period, we assessed HR, BP, and respiratory rate during a supine baseline period of at least $5 \mathrm{~min}$. We used IPG (11) continuously to measure resistance $\left(R^{0}\right)$ and beat-to-beat change in resistance $(\delta \mathrm{R})$ of thoracic, splanchnic, pelvic, and leg segments (explained above).

After supine data collections were complete, the LBNP group subjects were then subjected to stepwise increasing lower-body negative pressure $(-10,-20,-30,-40$, and $-50 \mathrm{mmHg})$ with $5 \mathrm{~min}$ at each pressure. The other group of subjects was tilted upright incrementally to $20^{\circ}, 40^{\circ}$, and $70^{\circ}$, remaining at each angle for $10 \mathrm{~min}$. Continuous $\mathrm{BP}, \mathrm{HR}$, and respirations were recorded; impedance flows and volumes were determined from electrical resistance measurements. For purposes of comparison, tabulated and graphic information represent data collected just before changes in LBNP pressure or tilt angle. Segmental flow data were tabulated before and throughout LBNP or HUT. 


\section{Data Analysis and Statistics}

Data were digitalized and stored in a computer and were analyzed off-line with custom software. HR and BP were determined at each stage expressed as means over 60-s intervals. Segmental blood volumes, flows, and peripheral resistances were calculated by formulas explained above. Time-series evaluations of raw data were analyzed by repeated-measures ANOVA. Results were calculated by using SPSS (Statistical Package for the Social Sciences) software version 14.0 and graphed using GraphPad prism (California) software version 4 . All tabular and graphic results are reported as means \pm SE.

\section{RESULTS}

All subjects completed $-50 \mathrm{mmHg}$ of LBNP and $70^{\circ}$ of HUT. No subject fainted or experienced presyncope that required early termination of testing.

\section{Baseline Hemodynamics}

Baseline hemodynamic characteristics of subjects undergoing LBNP and HUT protocols are shown in Table 1. SBP, DBP, MAP, and HR are not different between the groups.

\section{HR and BP during HUT and LBNP}

HR and BP changes are shown in Fig. 2. HR increased from $56 \pm 2$ to $62 \pm 4$ beats/min during LBNP and from $62 \pm 4$ to $96 \pm 6$ beats/min during HUT $(P<0.001)$. SBP and MAP were unchanged throughout LBNP and HUT. DBP increased from $72 \pm 1$ to $77 \pm 2 \mathrm{mmHg}$ during LBNP and from $67 \pm 2$ to $69 \pm 3 \mathrm{mmHg}$ during HUT. PP decreased from $51 \pm 3$ to $46 \pm 3 \mathrm{mmHg}$ during LBNP and from $53 \pm 4$ to $40 \pm 5 \mathrm{mmHg}$ in HUT $(P<0.01)$.

\section{IPG Changes in Segmental Blood Volumes and Blood Flows}

Similar changes in segmental blood flows in LBNP and HUT-As shown in Fig. 3, LBNP and HUT resulted in similar decreases in segmental blood flow over the negative pressures and angles tested. Impedance flows were expressed as percent changes. In both LBNP and HUT, there was a decrease in thoracic blood flow by $60 \%$, splanchnic blood by $40 \%$, pelvic blood flow by $40 \%$, and leg blood flow by $35 \%$.

Similar changes in segmental arterial resistances in LBNP and HUT-As shown in Fig. 4 there was an increase in arterial resistance $(P<0.001)$ in all four segments. These were similar for LBNP and HUT. Thus thoracic resistance increased by $\sim 145 \%$ for both LBNP and tilt; splanchnic resistance increased by $\sim 90 \%$ for both LBNP and tilt. Pelvic peripheral resistance increased by $90 \%$ following LBNP and $125 \%$ following HUT, and leg peripheral resistance increased $75 \%$ following LBNP and 140\% following HUT (Fig. 4, $P<$ $0.05)$.

\section{Similar changes in thoracic, pelvic, and leg blood volumes but directionally opposite changes in splanchnic blood volumes in LBNP and HUT-Changes in segmental blood volumes for LBNP and HUT are shown in Fig. 5. In both LBNP and HUT, thoracic volume decreased by $12 \%$, pelvic by $5 \%$, and leg by $8 \%(P<0.05)$. LBNP and HUT induced opposite volume shifts in splanchnic circulation. Splanchnic volume}


decreased in LBNP (12\%), although it increased in HUT (18\%; $P<0.001)$. Figure 6 highlights this directional difference between LBNP and HUT by depicting representative splanchnic blood volume changes in a representative subject undergoing LBNP and in a representative subject undergoing HUT.

\section{DISCUSSION}

Our main new finding is that the splanchnic reservoir fills (pools) during HUT and empties during lower-body negative pressure. The greater the tilt angle during HUT the greater the pooling. Conversely, the more negative the pressure during LBNP the greater the splanchnic emptying. Orthostatic stress is elicited by upright posture or standing. LBNP and HUT result in similar degrees of thoracic hypovolemia, resulting in unloading of the arterial and cardiopulmonary baroreflexes to a similar extent. This results in similar changes in regional blood flows and arterial resistances. However, the directionally opposite change in splanchnic volume is a fundamental dichot-omy between the two stressors.

Our investigation is unique in that it is the first to demonstrate that the progressive reduction in central blood volume induced by LBNP elicits a splanchnic emptying similar to that observed in hemorrhage. This finding provides new data that support the use of LBNP as an appropriate model to study the physiology of hemorrhage in humans.

The disparate findings can be explained by the effects of gravity. Splanchnic (e.g., portal) venous pressure is $\sim 12 \mathrm{mmHg}$ (6). From our data, $-50 \mathrm{mmHg}$ LBNP and $70^{\circ}$ HUT both produce a reduction in splanchnic blood flow of $\sim 40 \%$. Estimated central venous pressure supine is $\sim 6 \mathrm{mmHg}$ and upright at $70^{\circ} \sim 0 \mathrm{mmHg}$ (10). Estimated central venous pressure at $-50 \mathrm{mmHg}$ LBNP is also $\sim 0 \mathrm{mmHg}$ (29). For the sake of discussion, we partition the contributions to splanchnic venous pressure into a hemostatic component, the pressure at the central point of the splanchnic circulation (taken as mid-abdomen) resulting from gravity, and a dynamic or viscous component that arises as the pressure gradient required for venous return while supine. Assuming a reduction of splanchnic blood flow by $40 \%$ at an initial supine dynamic pressure of $12 \mathrm{mmHg}$ (hemostatic pressure), then a supine central venous pressure of $6 \mathrm{mmHg}$ implies that a pressure gradient of $12-6=6 \mathrm{mmHg}$ is needed on average to return splanchnic venous flow to the right heart. If venous resistance were to remain constant, then a $40 \%$ reduction would lead to a reduction of the gradient for splanchnic venous to $4.5 \mathrm{mmHg}$. This estimated gradient is not likely to be much smaller; if anything, the gradient is likely to be slightly larger because of venoconstriction. Because central venous pressure is reduced to $0 \mathrm{mmHg}$ during $-50 \mathrm{mmHg} \mathrm{LBNP}$, if gravity were absent, this implies a portal venous pressure of $\sim 4-5 \mathrm{mmHg}$. Assuming that this is equal to the average splanchnic venous pressure then the combined effects of elastic recoil from 12 to $4.5 \mathrm{mmHg}$ and splanchnic venoconstriction would produce considerable splanchnic emptying. This accounts for extensive emptying of the splanchnic vasculature during lowerbody negative pressure. To obtain a similar estimate of splanchnic emptying/pooling during upright tilt, we add the effects of gravity, i.e., add the hemostatic pressure to the dynamic pressure. By direct measurement, we estimate that the midpoint of the abdomen is $\sim 20 \mathrm{~cm}$ below the heart. At an angle of tilt of $70^{\circ}$, this corresponds to a hemostatic column of $\sin \left(70^{\circ}\right) \times 20 \mathrm{~cm}$ or $18.8 \mathrm{~cm}$. The conversion from centimeters of blood to millimeters 
mercury is $0.776 \times 18.8 \mathrm{~cm}=14.6 \mathrm{mmHg}$. Adding this to the dynamic pressure estimate yields a total splanchnic pressure of $14.5+4.5$ or $19 \mathrm{mmHg}$, which considerably exceeds estimated supine splanchnic venous pressure and accounts for splanchnic filling. One might predict that, for equivalent degrees of leg filling, there is less cardiovascular stress during LBNP than during HUT because of splanchnic emptying in the former and splanchnic filling in the latter.

The splanchnic circulation comprises the vessels of the liver, spleen, pancreas, and intestines. The pivotal role of the splanchnic circulation has been long appreciated (24). Specific organ blood reservoir capabilities vary across species (33). Thus, for example, Barcroft et al. (2) showed that splenic contraction could completely restore circulating blood volume in the dog during mild hemorrhage. The splanchnic circulation receives the largest blood flow of any regional circulation under resting conditions (36) and contains the largest blood volume (35). Its oxygen consumption is usually low (4). This allows for a great reduction in blood flow before organ ische-mia occurs (20). Active venoconstriction combined with elastic recoil of the veins greatly enhances the release of blood during circulatory insufficiency and specifically during hemorrhage (16, 34). Thus, Vatner (42) demonstrated that moderate hypotensive hemorrhage was associated with increased splanchnic arterial resistance and also peripheral vascular resistance, whereas severe shock was associated with further globally increased arterial resistance but nearly complete splanchnic emptying. Similar observations were made by Brunner et al (5), Shoukas et al. (37), Hainsworth and Karim (18), and Noble et al. (32) who attributed rapid splanchnic mobilization to sympathetic activation. Splanchnic emptying during hemorrhage also occurs in humans (22).

LBNP is an excellent model for hemorrhage (8) and produces unloading of the thoracic baroreceptors (3). The main advantage for LBNP over HUT in the study of hemorrhage is that it explicitly removes gravitational effects as a separate physiological stressor. Therefore, it is entirely expected that progressive stepwise splanchnic emptying should accompany stepwise decrements in LBNP. However, to our knowledge, we are the first to demonstrate this in humans and to show the large volumetric contribution to LBNP.

Although pooling during LBNP in diverse regional circulations has been studied, the splanchnic bed has not been specifically investigated. Thus Wolthuis et al. (47) suggested significant pooling in legs during $-40 \mathrm{mmHg}$ LBNP but did not consider splanchnic changes. White and Montgomery (43), using impedance methods, showed significant pelvic pooling in females but not in males during $50 \mathrm{mmHg}$ fixed LBNP. These investigators grouped the thoracic and splanchnic circulations together as the "trunk" and therefore could not discriminate specific splanchnic contributions.

HUT is an excellent model for standing or orthostatic stress (1) and produces unloading of the thoracic baroreceptors (9) that is qualitatively similar to LBNP or hemorrhage. However, orthostatic stress is driven by gravity. Thus dependent regional vasculature below the hemostatic indifferent point (23) will fill with blood while the vasculature above will empty. The indifference point in humans is regarded to be approximately at the xyphoid process. Thus, in our experiments, the splanchnic segment, which is demarcated by the xyphoid 
process above and the iliac crest below and is centered roughly at the umbilicus, will be subject to a net hemostatic pressure that produces filling. The indifference point may change with posture, with time, and with various fluid shifts out of (above the indifference point) and into (below the indifference point) the interstitial spaces. However, directional changes in segmental filling or emptying are fairly monotonic and are typically unaffected over the course of a typical HUT test. Thus, just as dependent legs fill with venous blood despite arterial vasoconstriction during upright tilt, so does the splanchnic bed fill with venous blood despite arterial vasoconstriction during upright tilt. It is possible that splanchnic venoconstriction could in part counteract this filling. Thus, in addition to passive elastic recoil resulting from arterial vasoconstriction, active splanchnic venoconstriction may contribute to emptying of the splanchnic venous system (34). However, by our estimation, splanchnic pressure is importantly increased during HUT, and it is not surprising that net venous filling occurs.

\section{Limitations}

Use of HR methods for ascertaining changes in segmental blood volumes has been debatable. There is ample precedence in its use in our laboratory $(38,40)$ and others $(28)$, although there is considerable biological variability.

The subjects were healthy young adults. Results are therefore not necessarily generalizable to all age ranges or to disease states.

The same subjects did not participate in both phases of study (LBNP and HUT protocols) and therefore could not act as their own controls. This was done to reduce the test duration in one single setting and improve subject compliance. A randomized counterbalanced study design would have an added advantage over the present design. However, the data are fairly tight with small variations even though patients were randomly selected. This strongly suggests that the phenomena observed are real and universal.

Also, the changes in stepwise incremental tilt used three steps compared with five steps of LBNP. However, these are standard protocols in which the overall duration of LBNP and HUT was designed to be similar. Thus the time taken for the regional fluid shifts is similar. Also, changes in vascular resistance and blood flows are similar in each group, suggesting that the schemes used for HUT and LBNP were similar from the standpoint of baroreflex unloading and vasoconstrictive response.

In conclusion, on the one hand, splanchnic blood pooling in HUT and directionally opposite splanchnic blood emptying in LBNP suggest an important difference caused by the effect of gravity on methods that are typically regarded as similar means to produce orthostatic stress. Although both produce similar changes in thoracic volume, thereby producing similar changes in thoracic baroreflex unloading, they cannot be regarded as completely similar stressors: upright tilt models standing or orthostatic stress; LBNP models hemorrhage or supine hypovolemic stress.

On the other hand, no method or model perfectly duplicates an actual condition. The data presented in the present paper support the notion that LBNP in fact duplicates the majority 
of regional circulatory responses that are induced by HUT. Splanchnic volume was the only response that was uniquely different. Thus the appropriateness of using LBNP to study orthostatic stress depends on the response of interest. For example, if one desires to investigate the relative (\%) effects of orthostatic stress on regional vascular resistances, it could be argued that LBNP is an appropriate model. Data acquired by these different techniques should be interpreted within this context.

\section{ACKNOWLEDGMENTS}

We thank members of the Department of Pediatrics, especially its Chairman, Dr. Leonard Newman, and the Division of Pediatric Cardiology, especially its Director, Dr. Michael H. Gewitz, for unflagging support. We also acknowledge our intellectual debt to our mentors Dr. Thomas H. Hintze, Dr. Gabor Kaley, Dr. David Robertson, and Dr. Phillip Low for constant inspiration and stimulation.

\section{GRANTS}

This work was supported by National Heart, Lung, and Blood Institute Grant 1R01HL-074873.

\section{REFERENCES}

1. Allen SC, Taylor CL, Hall VE. A study of orthostatic insufficiency by the tiltboard method. Am J Physiol. 1945; 143:11-20.

2. Barcroft J, Nisimaru Y, Puri SR. The action of the splanchnic nerves on the spleen. J Physiol. 1936; 87:189-197. [PubMed: 16994787]

3. Brown CM, Hecht MJ, Neundorfer B, Hilz MJ. Effects of lower body negative pressure on cardiac and vascular responses to carotid baroreflex stimulation. Physiol Res. 2003; 52:637-645. [PubMed: 14535840]

4. Brundin T, Wahren J. Whole body and splanchnic oxygen consumption and blood flow after oral ingestion of fructose or glucose. Am J Physiol Endocrinol Metab. 1993; 264:E504-E513.

5. Brunner MJ, Shoukas AA, MacAnespie CL. The effect of the carotid sinus baroreceptor reflex on blood flow and volume redistribution in the total systemic vascular bed of the dog. Circ Res. 1981; 48:274-285. [PubMed: 7460202]

6. Casey JJ, Lakey JR, Ryan EA, Paty BW, Owen R, O’Kelly K, Nanji S, Rajotte RV, Korbutt GS, Bigam D, Kneteman NN, Shapiro AM. Portal venous pressure changes after sequential clinical islet transplantation. Transplantation. 2002; 74:913-915. [PubMed: 12394830]

7. Coles DR, Kidd BS, Patterson GC. The reactions of the blood vessels of the human calf to increases in transmural pressure. J Physiol. 1956; 134:665-674. [PubMed: 13398951]

8. Cooke WH, Ryan KL, Convertino VA. Lower body negative pressure as a model to study progression to acute hemorrhagic shock in humans. J Appl Physiol. 2004; 96:1249-1261. [PubMed: 15016789]

9. Cooper VL, Hainsworth R. Carotid baroreceptor reflexes in humans during orthostatic stress. Exp Physiol. 2001; 86:677-681. [PubMed: 11571497]

10. Cui J, Durand S, Levine BD, Crandall CG. Effect of skin surface cooling on central venous pressure during orthostatic challenge. Am J Physiol Heart Circ Physiol. 2005; 289:H2429-H2433. [PubMed: 16024573]

11. Ebert TJ, Hughes CV, Tristani FE, Barney JA, Smith JJ. Effect of age and coronary heart disease on the circulatory responses to graded lower body negative pressure. Cardiovasc Res. 1982; 16:663-669. [PubMed: 7168843] 
12. El Bedawi KM, Hainsworth R. Combined head-up tilt and lower body suction: a test of orthostatic tolerance. Clin Auton Res. 1994; 4:41-47. [PubMed: 8054836]

13. Fu Q, Witkowski S, Okazaki K, Levine BD. Effects of gender and hypovolemia on sympathetic neural responses to orthostatic stress. Am J Physiol Regul Integr Comp Physiol. 2005; 289:R109_ R116. [PubMed: 15761188]

14. Geddes, LA.; Baker, LE. Principles of Applied Biomedical Instrumentation. Wiley; New York: 1989. Detection of physiological events by impedance; p. 594-600.

15. Geddes LA, Sadler C. The specific resistance of blood at body temperature. Med Biol Eng. 1973; 11:336-339. [PubMed: 4746389]

16. Granger DN, Stokes KY, Shigematsu T, Cerwinka WH, Tailor A, Krieglstein CF. Splanchnic ischaemia-reperfusion injury: mechanistic insights provided by mutant mice. Acta Physiol Scand. 2001; 173:83-91. [PubMed: 11678730]

17. Greenway CV, Lister GE. Capacitance effects and blood reservoir function in the splanchnic vascular bed during non-hypotensive haemorrhage and blood volume expansion in anaesthetized cats. J Physiol. 1974; 237:279-294. [PubMed: 4825450]

18. Hainsworth R, Karim F. Changes in abdominal vascular capacity in response to stimulation of efferent sympathetic nerves. J Physiol. 1974; 239:26P-27P.

19. Halliwill JR, Lawler LA, Eickhoff TJ, Joyner MJ, Mulvagh SL. Reflex responses to regional venous pooling during lower body negative pressure in humans. J Appl Physiol. 1998; 84:454458. [PubMed: 9475852]

20. Heino A, Hartikainen J, Merasto ME, Koski EM, Alhava E, Takala J. Systemic and regional effects of experimental gradual splanchnic ischemia. J Crit Care. 1997; 12:92-98. [PubMed: 9165417]

21. Hughson RL, Maillet A, Gharib C, Fortrat JO, Yamamoto Y, Pavy-Letraon A, Riviere D, Guell A. Reduced spontaneous baroreflex response slope during lower body negative pressure after 28 days of head-down bed rest. J Appl Physiol. 1994; 77:69-77. [PubMed: 7961277]

22. Jakob SM. Clinical review: splanchnic ischaemia. Crit Care. 2002; 6:306-312. [PubMed: 12225604]

23. Kirsch K, Merke J, Hinghofer-Szalkay H. Fluid volume distribution within superficial shell tissues along body axis during changes of body posture in man: the application of a new miniature plethysmographic method. Pflugers Arch. 1980; 383:195-201. [PubMed: 7190673]

24. Kitano A, Shoemaker JK, Ichinose M, Wada H, Nishiyasu T. Comparison of cardiovascular responses between lower body negative pressure and head-up tilt. J Appl Physiol. 2005; 98:20812086. [PubMed: 15761089]

25. Montgomery LD. Body volume changes during simulated microgravity I: Technique and comparison of men and women during horizontal bed rest. Aviat Space Environ Med. 1993; 64:893-898. [PubMed: 8240192]

26. Montgomery LD. Body volume changes during simulated microgravity.II. Comparison of horizontal and head-down bed rest. Aviat Space Environ Med. 1993; 64:899-904. [PubMed: 8240193]

27. Montgomery LD, Hanish HM, Marker RA. An impedance device for study of multisegment hemodynamic changes during orthostatic stress. Aviat Space Environ Med. 1989; 60:1116-1122. [PubMed: 2818406]

28. Montgomery LD, Kirk PJ, Payne PA, Gerber RL, Newton SD, Williams BA. Cardiovascular responses of men and women to lower body negative pressure. Aviat Space Environ Med. 1977; 48:138-145. [PubMed: 871283]

29. Mosqueda-Garcia R, Furlan R, Fernandez-Violante R, Desai T, Snell M, Jarai Z, Ananthram V, Robertson RM, Robertson D. Sympathetic and baroreceptor reflex function in neurally mediated syncope evoked by tilt. J Clin Invest. 1997; 99:2736-2744. [PubMed: 9169504]

30. Murray RH, Thompson LJ, Bowers JA, Albright CD. Hemodynamic effects of graded hypovolemia and vasodepressor syncope induced by lower body negative pressure. Am Heart J. 1968; 76:799-811. [PubMed: 5721838]

31. Musgrave FS, Zechman FW, Mains RC. Changes in total leg volume during lower body negative pressure. Aerosp Med. 1969; 40:602-606. [PubMed: 5786684] 
32. Noble BJ, Drinkhill MJ, Myers DS, Hainsworth R. Reflex control of splanchnic blood volume in anaesthetized dogs. J Physiol. 1998; 513:263-272. [PubMed: 9782176]

33. Rothe, CF. The Cardiovascular System: Peripheral Circulation and Organ Blood Flow. Handbook of Physiology. Vol. III. Am Physiol Soc; Bethesda, MD: 1983. Venous system: physiology of the capacitance vessels; p. 397-452.sect. 22pt. 1

34. Rothe CF. Reflex control of veins and vascular capacitance. Physiol Rev. 1983; 63:1281-1342. [PubMed: 6361810]

35. Rowell, LB. Human Cardiovascular Control. Oxford Univ Press; New York, NY: 1993.

36. Rowell LB. Reflex control of regional circulations in humans. J Auton Nerv Syst. 1984; 11:101114. [PubMed: 6386940]

37. Shoukas AA, MacAnespie CL, Brunner MJ, Watermeier L. The importance of the spleen in blood volume shifts of the systemic vascular bed caused by the carotid sinus baroreceptor reflex in the dog. Circ Res. 1981; 49:759-766. [PubMed: 7261270]

38. Stewart JM, Medow MS, Glover JL, Montgomery LD. Persistent splanchnic hyperemia during upright tilt in postural tachycardia syndrome. Am J Physiol Heart Circ Physiol. 2006; 290:H665H673. [PubMed: 16143646]

39. Stewart JM, Montgomery LD. Regional blood volume and peripheral blood flow in postural tachycardia syndrome. Am J Physiol Heart Circ Physiol. 2004; 287:H1319-H1327. [PubMed: 15117717]

40. Stewart JM, Montgomery LD, Glover JL, Medow MS. Changes in regional blood volume and blood flow during static handgrip. Am J Physiol Heart Circ Physiol. 2007; 292:H215-H223. [PubMed: 16936003]

41. Stewart JM, Weldon A. Contrasting neurovascular findings in chronic orthostatic intolerance and neurocardiogenic syncope. Clin Sci. 2003; 104:329-340. [PubMed: 12653674]

42. Vatner SF. Effects of hemorrhage on regional blood flow distribution in dogs and primates. J Clin Invest. 1974; 54:225-235. [PubMed: 4211160]

43. White DD, Montgomery LD. Pelvic blood pooling of men and women during lower body negative pressure. Aviat Space Environ Med. 1996; 67:555-559. [PubMed: 8827137]

44. Wolthuis RA, Hoffler GW, Johnson RL. Lower body negative pressure as an assay technique for orthostatic tolerance. II. A comparison of the individual response to incremental vs constant levels of LBNP. Aerosp Med. 1970; 41:419-424. [PubMed: 5439850]

45. Wolthuis RA, Hoffler GW, Johnson RL. Lower body negative pressure as an assay technique for orthostatic tolerance 3: a comparison of the individual response to incremental leg negative pressure vs incremental lower body negative pressure. Aerosp Med. 1970; 41:1354-1357. [PubMed: 5482606]

46. Wolthuis RA, Hoffler GW, Johnson RL. Lower body negative pressure as an assay technique for orthostatic tolerance. I. The individual response to a constant level $(-40 \mathrm{mmHg})$ of LBNP. Aerosp Med. 1970; 41:29-35. [PubMed: 5443044]

47. Wolthuis RA, LeBlanc A, Carpentier WA, Bergman SA Jr. Response of local vascular volumes to lower body negative pressure stress. Aviat Space Environ Med. 1975; 46:697-702. [PubMed: 1131133] 
A

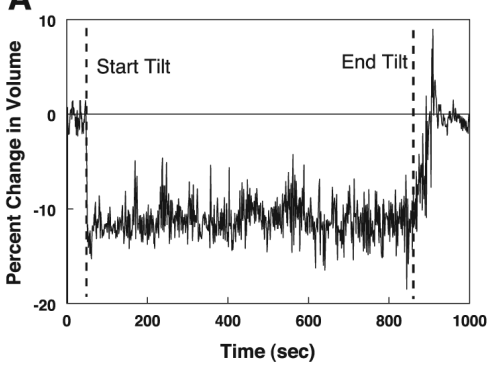

C

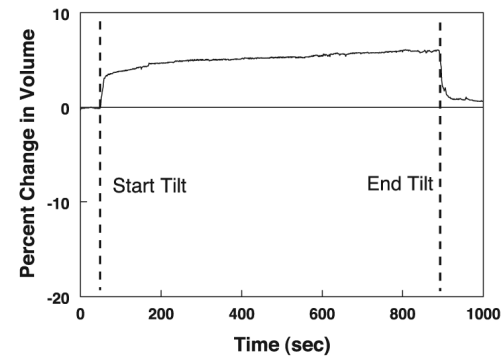

B

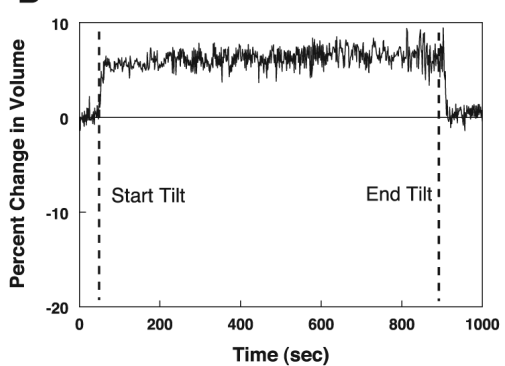

D

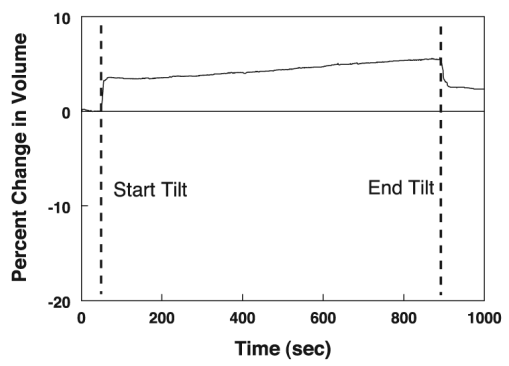

Fig. 1.

Changes in thoracic (A), splanchnic (B), pelvic (C), and leg blood (D) volumes during headup tilt table testing to $70^{\circ}$, calculated from impedance plethysmography measurements (unpublished observations). The thorax empties while all other regional circulations fill. 

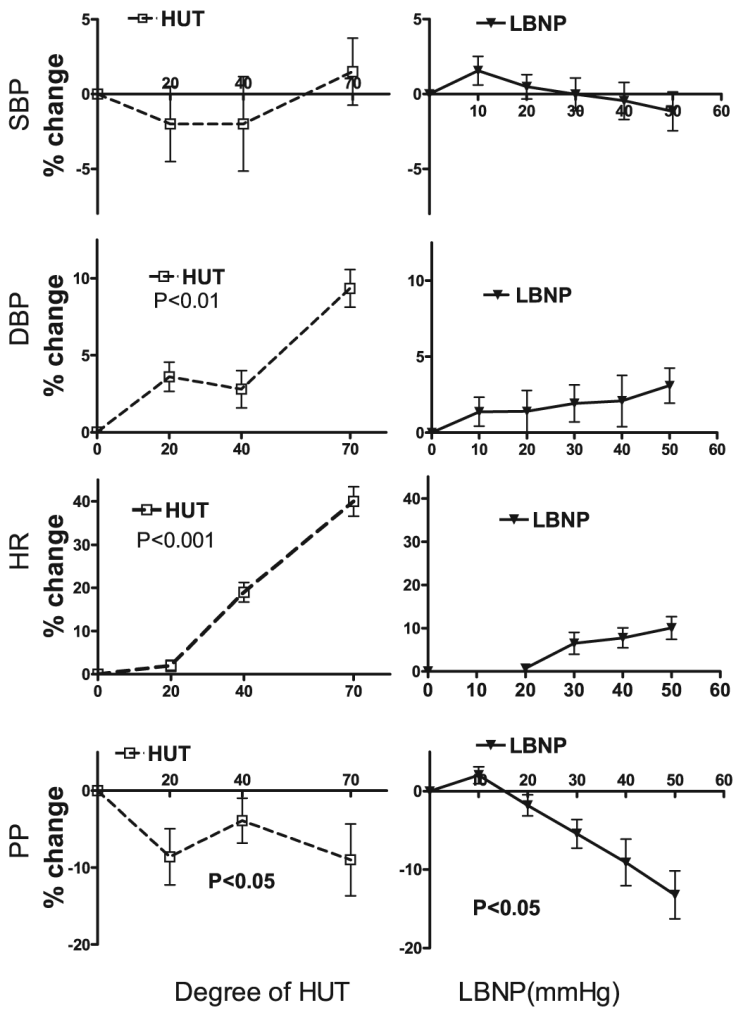

Fig. 2.

Hemodynamic changes following head-up tilt (HUT) and lower body negative pressure (LBNP). Left: \%changes in HUT. Right: \%changes in LBNP. SBP, systolic blood pressure (mmHg); DBP, diastolic blood pressure (mmHg); HR, heart rate (beats/min); PP, pulse pressure (mmHg). Following HUT and LBNP, the SBP was maintained, whereas HR increased and PP decreased $(P<0.05)$. 

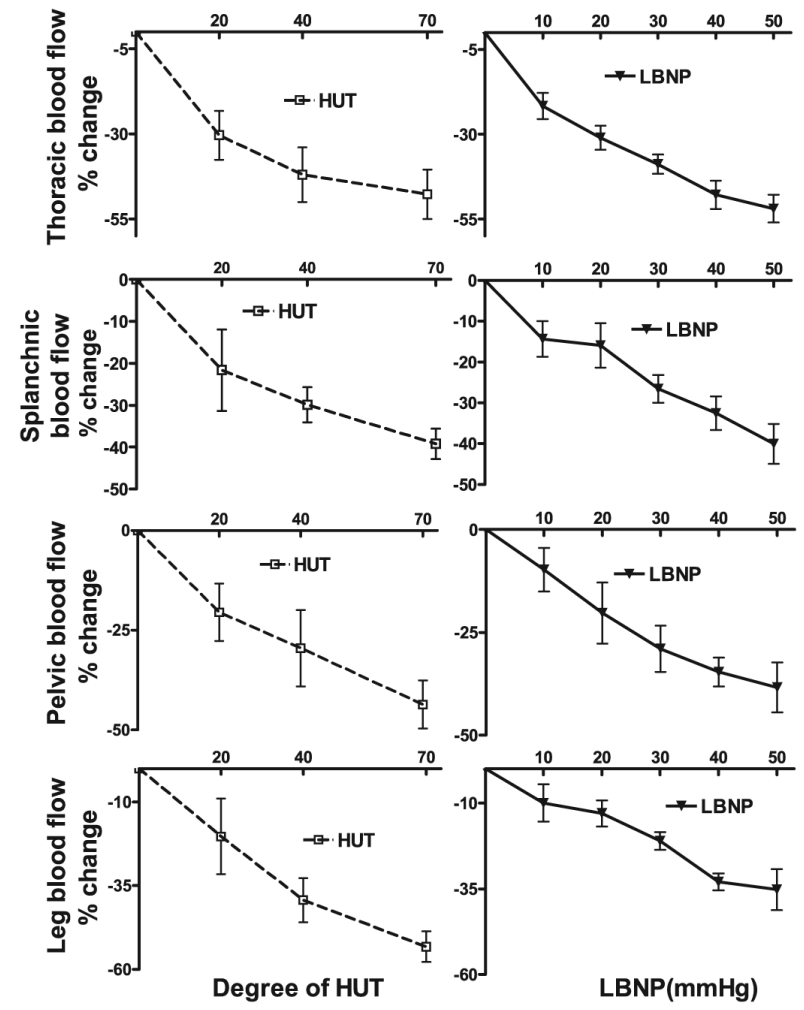

Fig. 3.

Segmental blood flow changes following HUT and LBNP. Left: \%changes of blood flow in HUT. Right: \%changes in LBNP in thoracic (top), splanchnic (2nd from top), pelvic (3rd from top), and leg (bottom). There is a generalized reduction in blood flow in all compartments. All the changes were significant $(P<0.05)$ compared with baseline. 

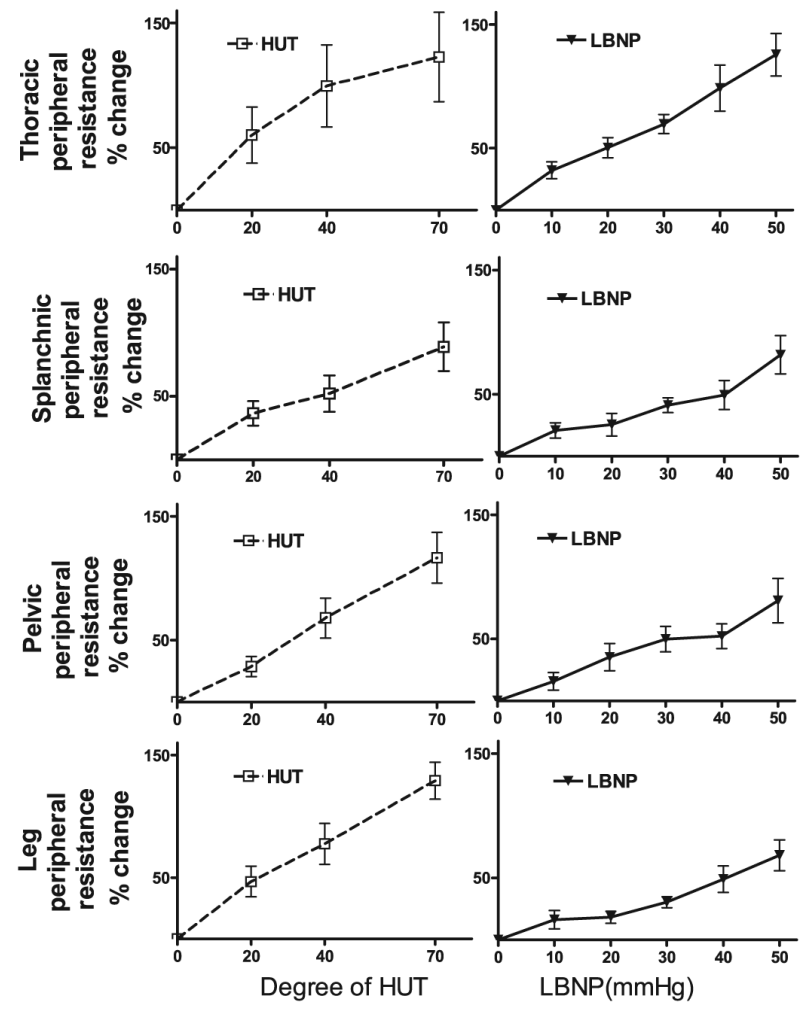

Fig. 4.

Segmental arterial resistance changes following HUT and LBNP. Left: \%changes of peripheral arterial resistance in HUT. Right: \%changes of peripheral arterial resistance in LBNP in respective regional segments. Note the generalized increase in peripheral arterial resistance in all compartments. All the changes were significant $(P<0.05)$. 

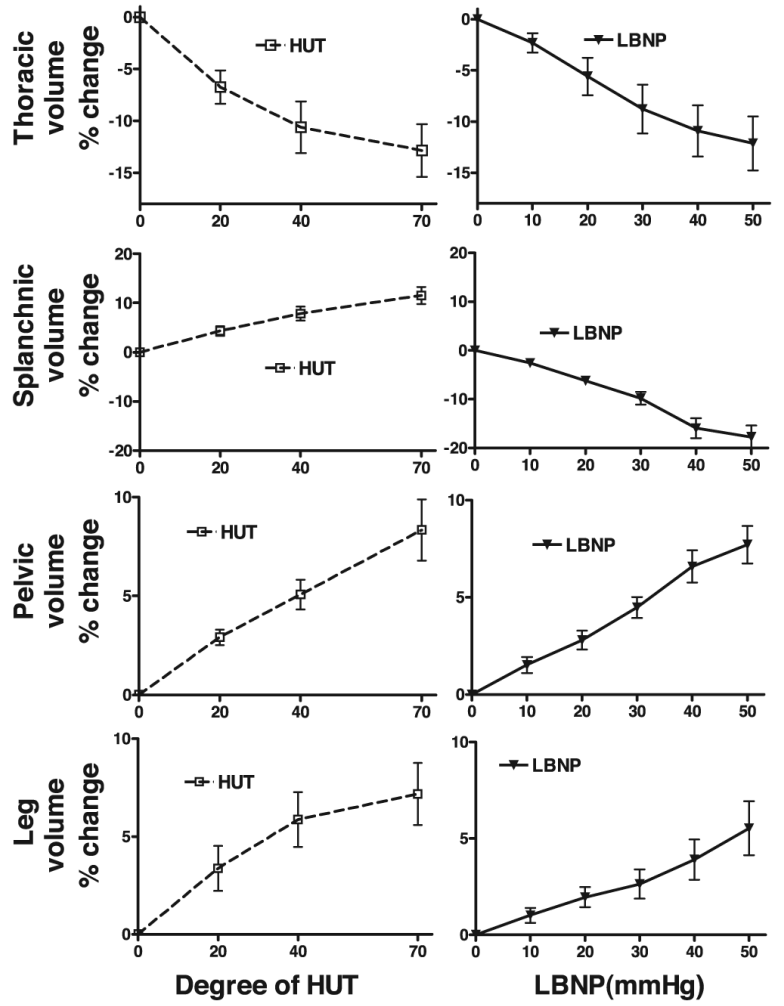

Fig. 5.

Segmental blood volume changes following HUT and LBNP. Left: \%changes of blood volume in HUT. Right: \%changes of blood volume in LBNP in respective regional segments. Note the directionally opposite volume changes in splanchnic compartment. All the changes were significant $(P<0.05)$. 


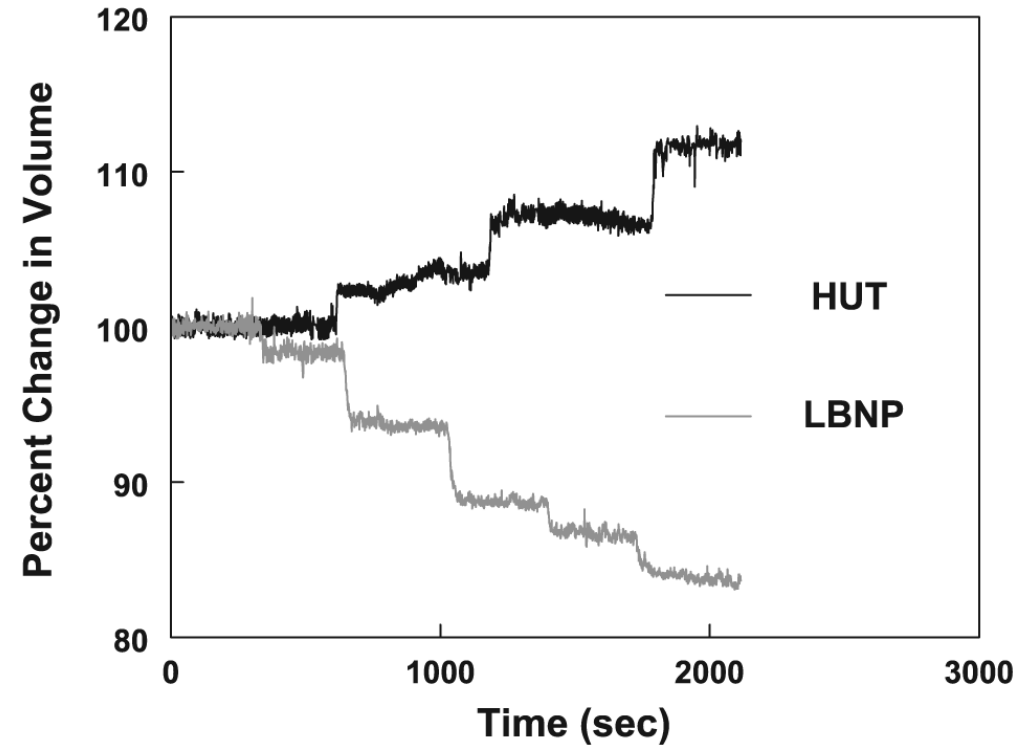

Fig. 6.

Representative tracings showing fractional volume shifts in splanchnic regional circulation in a representative subject. HUT is shown in black, whereas LBNP is shown in gray. Note progressive splanchnic filling in HUT and splanchnic emptying in LBNP. 


\section{Table 1}

Baseline demographics and hemodynamics

\begin{tabular}{lcc}
\hline \multicolumn{1}{c}{ Parameters } & LBNP $(\boldsymbol{n}=\mathbf{1 0})$ & HUT $(\boldsymbol{n}=\mathbf{8})$ \\
\hline Age, yrs & $24.6 \pm 0.6$ & $20 \pm 1$ \\
Gender, \%female & 70 & 62.2 \\
Resting SBP, mmHg & $122.3 \pm 4$ & $120 \pm 3$ \\
Resting DBP, mmHg & $72 \pm 2$ & $67 \pm 3$ \\
Resting MAP, mmHg & $87 \pm 3$ & $85 \pm 3$ \\
Resting HR, beats/min & $56 \pm 2$ & $62 \pm 2$ \\
\hline
\end{tabular}

Results are represented as means \pm SE; $n$, no. of subjects. LBNP, lower body negative pressure; HUT, head-up tilt; SBP, systolic blood pressure; $\mathrm{DBP}$, diastolic blood pressure; MAP, mean arterial pressure; HR, heart rate. 